\title{
A STUDY OF DENSITY MODULATION INDEX IN THE INNER HELIOSPHERIC SOLAR WIND DURING SOLAR CYCLE 23
}

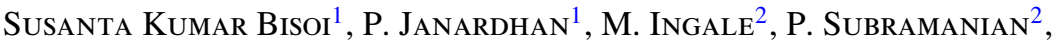 \\ S. ANANTHAKRISHNAN ${ }^{3}$, M. TOKUMARU ${ }^{4}$, AND K. FuJiki ${ }^{4}$ \\ ${ }^{1}$ Astronomy and Astrophysics Division, Physical Research Laboratory, Ahmedabad 380009, India; susanta@prl.res.in, jerry@prl.res.in \\ ${ }^{2}$ Indian Institute of Science Education and Research, Dr. Homi Bhabha Road, Pashan, Pune 411021, India; \\ i.madhusudan@students.iiserpune.ac.in,p.subramanian@iiserpune.ac.in \\ ${ }^{3}$ Department of Electronic Science, University of Pune, Pune 411007, India; subra.anan@ gmail.com \\ ${ }^{4}$ Solar-Terrestrial Environment Laboratory, Nagoya University, Nagoya 464-8601, Japan; \\ tokumaru@stelab.nagoya-u.ac.jp, fujiki@stelab.nagoya-u.ac.jp \\ Received 2014 May 27; accepted 2014 August 17; published 2014 October 14
}

\begin{abstract}
The ratio of the rms electron density fluctuations to the background density in the solar wind (density modulation index, $\left.\epsilon_{N} \equiv \Delta N / N\right)$ is of vital importance for understanding several problems in heliospheric physics related to solar wind turbulence. In this paper, we have investigated the behavior of $\epsilon_{N}$ in the inner heliosphere from 0.26 to $0.82 \mathrm{AU}$. The density fluctuations $\Delta N$ have been deduced using extensive ground-based observations of interplanetary scintillation at $327 \mathrm{MHz}$, which probe spatial scales of a few hundred kilometers. The background densities $(N)$ have been derived using near-Earth observations from the Advanced Composition Explorer. Our analysis reveals that $0.001 \lesssim \epsilon_{N} \lesssim 0.02$ and does not vary appreciably with heliocentric distance. We also find that $\epsilon_{N}$ declines by $8 \%$ from 1998 to 2008 . We discuss the impact of these findings on problems ranging from our understanding of Forbush decreases to the behavior of the solar wind dynamic pressure over the recent peculiar solar minimum at the end of cycle 23 .
\end{abstract}

Key words: interplanetary medium - solar wind - turbulence

Online-only material: color figures

\section{INTRODUCTION}

The solar wind is an unparalleled natural laboratory for the study of magneto-hydrodynamic turbulence (e.g., Tu \& Marsch 1995; Goldstein et al. 1995; Bruno \& Carbone 2005; Marsch 2006; Spangler 2009). It involves fluctuations in magnetic field, density, and velocity over a wide range of spatial and temporal scales. Turbulent density fluctuations in the solar wind have been observed over heliocentric distances ranging from $\sim 0.14 \mathrm{AU}$ or $30 R_{\odot}$ to $1 \mathrm{AU}$ or $215 R_{\odot}$ from the Sun, where $R_{\odot}$ is the solar radius (Coles 1978; Marsch \& Tu 1990; Bavassano \& Bruno 1995; Janardhan et al. 1996; Efimov et al. 2000; Spangler 2002; Bird et al. 2003; Spangler 2009; Tokumaru et al. 2012). Moreover, density fluctuations are often believed to be better tracers of solar wind flows as compared to solar wind density (Ananthakrishnan et al. 1980; Woo et al. 1995; Huddleston et al. 1995). Detailed measurements of solar wind density fluctuations near the Earth have been made using in situ data from spacecraft such as Helios 1, Helios 2, Wind, and Ulysses.

MHD turbulence theory generally assumes incompressibility, and density fluctuations do not fit into the narrative. Furthermore, the scaling law in (spatial) wavenumber space exhibited by density turbulence observations is generally consistent with the Kolmogorov theory, which in fact holds for incompressible fluid turbulence in the absence of magnetic fields. The implications of compressibility (as evidenced by observations of turbulent density fluctuations) via theories of MHD turbulence is a subject of considerable discussion (Tu \& Marsch 1994; Hnat et al. 2005; Shaikh \& Zank 2010). In particular, knowing the manner in which the density modulation index,

$$
\epsilon_{N} \equiv \frac{\Delta N}{N},
$$

varies with distance from the Sun is of vital importance for a variety of applications.

In the expression for $\epsilon_{N}$ (Equation (1)), the quantity $\Delta N$ represents the turbulent density fluctuation while $N$ is the background density. An understanding of $\epsilon_{N}$ is important for understanding turbulent dissipation and consequent local heating of the solar wind (Carbone et al. 2009). It is also an important ingredient in constructing models for the quantity $C_{N}^{2}$, which is the amplitude of the density turbulence spectrum (Thejappa \& MacDowall 2008). In turn, $C_{N}^{2}$ is crucial for understanding angular broadening of radio sources due to solar wind turbulence (Janardhan \& Alurkar 1993; Bastian 1994; Subramanian \& Cairns 2011) and for explaining the rather low brightness temperatures of the solar corona at meter to decameter wavelengths (Thejappa \& MacDowall 2008). A crucial role is also played by $\epsilon_{N}$ in influencing the propagation of energetic electrons, produced by solar flares and other explosive solar surface phenomena, through the heliosphere (Reid \& Kontar 2010).

Recently, using interplanetary scintillation (IPS) measurements of scintillation index from 1983 to 2009, the solar wind micro-turbulence levels in the inner heliosphere were shown to be steadily declining since $\approx 1995$ (Janardhan et al. 2011). Using ground-based magnetograms from the National Solar Observatory at Kitt Peak, a steady and systematic decline in solar polar fields, starting from $\approx 1995$, has also been reported (Janardhan et al. 2010; Bisoi et al. 2014). In addition, both in-ecliptic (Advanced Composition Explorer (ACE) and Wind; Jian et al. 2011) and out-of-ecliptic (Ulysses; McComas et al. 2008) solar wind measurements, during the recent minimum of solar cycle 23 , in 2008 and 2009, have shown a reduction in solar wind dynamic pressure of about $20 \%$. Under these very unusual and unique 
circumstances of declining solar polar field strengths and density turbulence levels $(\propto \Delta \mathrm{N}$; Janardhan et al. 2010, 2011; Bisoi et al. 2014), studies of the temporal changes of $\epsilon_{N}$ in the inner heliosphere are both important and crucial for understanding the relation between magnetic field fluctuations and density fluctuations. Such a study also impinges on the important question of the role of the dynamic pressure exerted by the solar wind on Earth's magnetosphere during this unusual phase.

The first measurements of $\epsilon_{N}$ were made at heliocentric distances $\lesssim 40 R_{\odot}$, by Woo et al. (1995) using Ulysses measurements obtained in 1991. Subsequently, density fluctuations in different types of solar wind flows have been reported at $1 \mathrm{AU}$ (Huddleston et al. 1995) and also in the region from 0.3 to $1 \mathrm{AU}$ using the Helios 2 spacecraft, interplanetary plasma data, obtained with a time cadence of 45 minutes (Bavassano \& Bruno 1995). These authors reported a $\epsilon_{N}$ of $\approx 0.1$ and proposed that compressive phenomena were not strong enough at the 45 minute cadence used for the observations. Further, Spangler (2002) reported a $0.06 \lesssim \epsilon_{N} \lesssim 0.15$ in the heliocentric distance range $16-26 R_{\odot}$. Using Wind spacecraft data at 1 AU, Spangler $\&$ Spitler (2004) have estimated $\epsilon_{N}$ of the order of 0.03-0.08 and proposed both a linear and quadratic relationship between the $\epsilon_{N}$ and the magnetic field index $\left(\epsilon_{B}\right)$ in regions of the nearSun solar wind. The data used in previous papers have been sparse, with either the observations being confined to a small region of the heliosphere or covering periods from a few days to years. However, in this paper, we have made use of observations spanning the entire inner heliosphere covering the heliocentric distance range of $0.26-0.82 \mathrm{AU}$ corresponding to $55-175 R_{\odot}$. In addition, our data set of 11 years covers the entirety of solar cycle 23 , thereby enabling a study of the long-term temporal variation in $\epsilon_{N}$ as well.

In this study, we have made use of extensive and systematic IPS measurements to investigate the radial evolution of $\epsilon_{N}$ defined in Equation (1). While electron density fluctuations have been estimated at $327 \mathrm{MHz}$ using measurements from the multistation IPS observatory of the Solar-Terrestrial Environment Laboratory (STEL), Japan, solar wind densities used were derived from in situ observations from the $A C E$ spacecraft (Stone et al. 1998) with $\epsilon_{N}$ being estimated for the period 1998-2008, covering all of solar cycle 23 .

The rest of the paper is organized as follows. Section 2 briefly discusses IPS as well as phase modulation of plane waves by the solar wind. In Section 3, the use of IPS and $A C E$ data and their analyses are discussed. Subsequently, in Section 4 we verify the long term temporal and spatial behavior of $\epsilon_{N}$. Finally, Section 5 summarizes our results.

\section{INTERPLANETARY SCINTILLATION}

IPS is a diffraction phenomenon in which coherent electromagnetic radiation from a distant radio source passes through the turbulent and refracting solar wind and suffers scattering. This results in random temporal variations of the signal intensity (scintillation) at the Earth. A schematic illustration of the typical IPS observing geometry is shown in Figure 1. The dashed lines in Figure 1 lie in the ecliptic plane, while the solid lines lie out of the ecliptic plane. The long-dashed line is the orbit of the Earth around the Sun. The line of sight (LOS) to a distant compact radio source with respect to the Sun ("S") and the Earth ("E") is shown by a solid line from E passing through the point "P," the point of closest approach of the LOS to the Sun. The angles $\epsilon$ and $\gamma$ are, respectively, the solar elongation and heliographic latitude of the source while " $\mathrm{A}$ " is the foot point of a perpendicu-

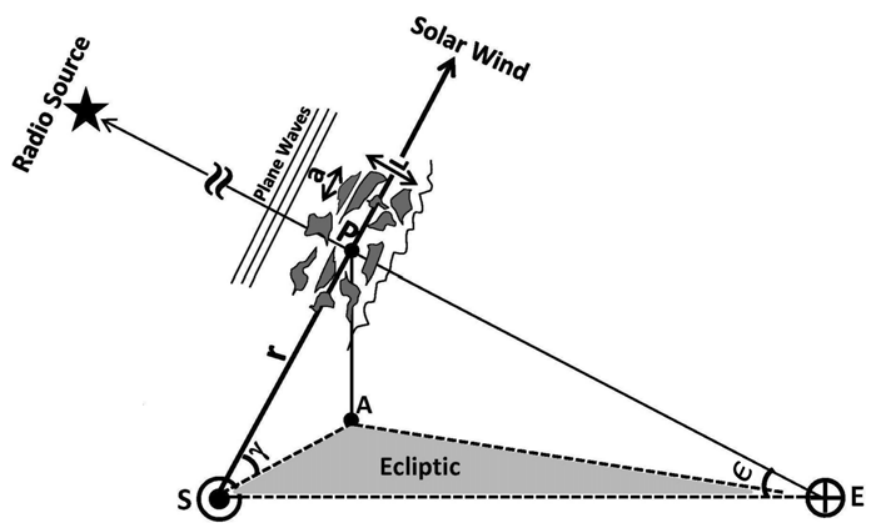

Figure 1. Schematic illustration of the IPS observing geometry. The Earth, the Sun, the point of closest approach of the LOS to the Sun, and the foot point of a perpendicular line from $\mathrm{P}$ to the ecliptic plane are shown by points $\mathrm{E}, \mathrm{S}, \mathrm{P}$, and A while the angles $\epsilon$ and $\gamma$ are the solar elongation and heliographic latitude of the observed source.

lar line from $P$ to the ecliptic plane. The heliocentric distance " $r$ " of the radio source, in astronomical units, is given by $r=\sin (\epsilon)$. It must be noted that the scintillations observed on Earth are modulated by the Fresnel filter function $\operatorname{Sin}^{2}\left(q^{2} \lambda z / 4 \pi\right)$, where $q$ is the wave number of the irregularities, $z$ is the distance from $\mathrm{E}$ to $\mathrm{P}$, and $\lambda$ is the observing wavelength. Due to the action of the Fresnel filter, IPS observations at $327 \mathrm{MHz}$ enable one to probe solar wind electron density fluctuations of scale sizes $\leqslant 1000 \mathrm{~km}$ both in and out of the ecliptic (Pramesh Rao et al. 1974; Coles \& Filice 1985; Yamauchi et al. 1998; Fallows et al. 2008) and over a wide range of heliocentric distances in the inner heliosphere (Janardhan et al. 1996).

Besides density fluctuations of spatial scale sizes $\leqslant 10^{3} \mathrm{~km}$, there are large-scale solar wind density fluctuations caused by structures such as coronal mass ejections (CMEs) and solar flares, which originate on the solar surface. The typical scale sizes of these structures range from $10^{4}$ to $10^{7} \mathrm{~km}$. The action of the Fresnel filter for scale sizes $\geqslant 10^{3} \mathrm{~km}$ is such that it will give rise to scintillation at distances $>1 \mathrm{AU}$, or, in other words, the Earth would be well within the Fresnel or near zone for these scale sizes. The IPS phenomenon therefore has a built in filter that makes it insensitive to contributions from large-scale size density irregularities. In fact, this property of IPS has even been exploited to study the fine-scale structure in cometary ion tails during radio source occultations by cometary tail plasma (Ananthakrishnan et al. 1975; Janardhan et al. 1991, 1992).

The degree to which compact, point-like, extragalactic radio sources exhibit scintillation, as observed by ground-based radio telescopes, is quantified by the scintillation index $(m)$ given by $m=\Delta \mathrm{S} /\langle S\rangle$, where $\Delta \mathrm{S}$ is the scintillating flux and $\langle S\rangle$ is the mean flux of the radio source being observed. For a given IPS observation, $m$ is simply the rms deviation of the signal intensity to the mean signal intensity and can be easily determined from the observed intensity fluctuations of compact extragalactic radio sources.

Though IPS measures only small-scale fluctuations in density and not the bulk density itself, it has been shown (Hewish et al. $1985)$ that there were no variations in IPS measurements of $\Delta N$ that were not associated with corresponding variations in density $N$. These authors used a normalized scintillation index " $g$ " (a good proxy for the density) to derive a relation between " $g$," and the density given by $g=\left(\mathrm{N} \mathrm{cm}^{-3} / 9\right)^{0.52 \pm 0.05}$.

For an ideal point-like radio source and at an observing wavelength $\lambda, m$ will steadily increase with decreasing distance 
" $r$ " from the Sun until it reaches a value of unity at some distance from the Sun. As $r$ continues to decrease beyond this point, $m$ will again drop off to values below unity. This turnover distance is a function of observing frequency and at $327 \mathrm{MHz}(\lambda=$ $92 \mathrm{~cm}$ ) occurs at $\approx 0.2 \mathrm{AU}$ or $\approx 40 R_{\odot}$. The region beyond the turn-over distance is known as the weak scattering regime. In addition to the dependence on heliocentric distance, $m$ will also reduce with an increase in the angular diameter of the radio source being observed.

\subsection{Phase Modulations of Waves and Scintillation Index}

The assumption that the solar wind is considered to be confined to a thin slab as depicted in Figure 1 is due to the fact that the solar wind scattering function $\beta(r) \propto r^{-4}$. Hence, most of the contribution to the scintillation will come from the point " $\mathrm{P}$ " on the LOS that is closest to the sun. Plane waves from distant, compact extragalactic radio sources on passing through the thin slab of density irregularities will have an rms phase deviation $\left(\phi_{\mathrm{rms}}\right)$ imposed across their wave fronts. The expression for $\phi_{\mathrm{rms}}$ is

$$
\phi_{\mathrm{rms}}=(2 \pi)^{\frac{1}{4}} \lambda r_{e}(a L)^{\frac{1}{2}}\left[<\Delta N^{2}>\right]^{\frac{1}{2}},
$$

where $r_{e}$ is the classical electron radius, $\lambda$ is the observing wavelength, and $a$ is the typical scale size in the thin screen of thickness $L$ (see Figure 1). In the weak scattering regime, $m$ is given by

$$
m \approx \sqrt{2} \phi_{\mathrm{rms}} .
$$

Equations (2) and (3) can be rewritten as

$$
\Delta N=\frac{m}{(2)^{\frac{1}{2}}(2 \pi)^{\frac{1}{4}} \lambda r_{e}(a L)^{\frac{1}{2}}} .
$$

Equation (4) gives us a prescription for determining the quantity $\Delta N$ from observations of $m$.

\section{DATA ANALYSIS}

Regular IPS observations on a set of about 200 chosen extragalactic radio sources have been carried out to determine solar wind velocities and scintillation indices at $327 \mathrm{MHz}$ (Kojima \& Kakinuma 1990; Asai et al. 1998) since 1983 at the multi-station IPS observatory of STEL, Japan. Prior to 1994, these observations were carried out by the three-station IPS facility at Toyokawa, Fuji, and Sugadaira. In 1994, one more antenna was commissioned at Kiso forming a four-station dedicated IPS network that has been making systematic and reliable estimates of solar wind velocities and scintillation indices (Tokumaru et al. 2012) except for a data gap of one year in 1994. Systematic observations have been carried out on about a dozen selected radio sources each day such that each source would have been observed over the whole range of heliocentric distances between 0.2 and $0.8 \mathrm{AU}$ in a period of about 1 yr. We have employed the daily measurements of $m$, spanning the period from 1998 to 2008 , covering solar cycle 23 .

Very compact radio sources are extremely rare and it has been established at a number of frequencies, using both IPS (Bourgois 1969; Bourgois \& Creynet 1972; Milne 1976) and long baseline interferometry (Clark et al. 1968; Clarke et al. 1969 ), that the radio source 1148-001 has an angular diameter of $\approx 10$ milliarcsecond (mas) at meter wavelengths. Thus, the source 1148-001 can be treated as a nearly ideal point source at $327 \mathrm{MHz}$, with almost all of its flux contained in a compact

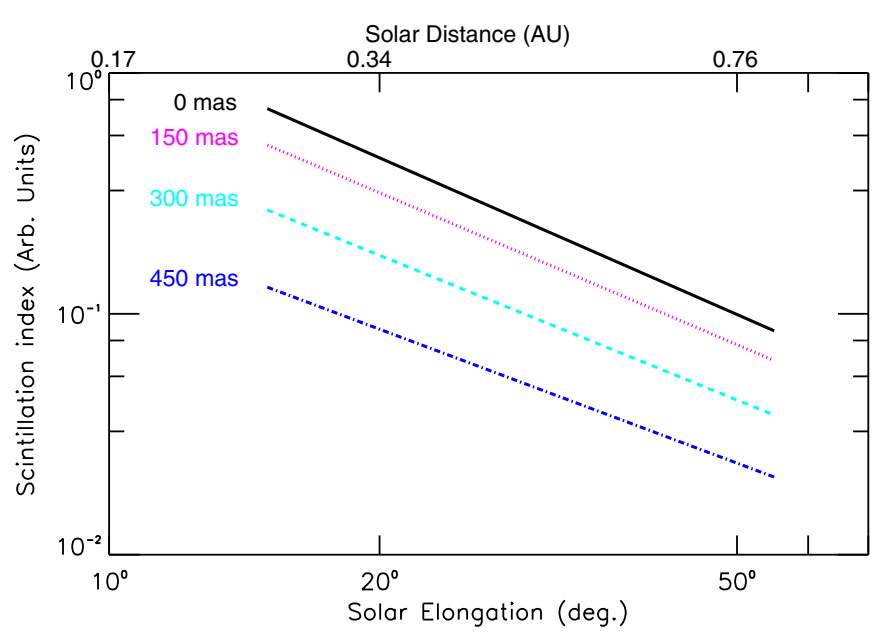

Figure 2. Curves of theoretically values of $m$ as a function of solar elongation for various source sizes corresponding to sizes of 0 mas, 150 mas, 300 mas, and 450 mas. These theoretical values of $m$ are computed using the Marians (1975) model.

(A color version of this figure is available in the online journal.)

scintillating component with very little flux outside this compact component (Swarup 1977; Venugopal et al. 1985). As stated earlier, for such ideal point sources, $m$ will be unity at the turnover distance, and will decrease as the distance of the LOS to the source moves further away from the Sun. For sources with larger angular diameters, $m$ will be less than unity at the turn-over distance.

Marians (1975) computed values of $m$ for radio sources of a given source size as a function of $r$ by obtaining theoretical temporal power spectra using a standard solar wind model assuming weak scattering and a power-law distribution of density irregularities in the IP medium. Figure 2 shows curves of theoretical $m$, computed using the Marians model (Marians 1975), as a function of $\epsilon$ (in degrees) for source sizes of 0 mas, 150 mas, 300 mas, and 450 mas, respectively. All the curves are plotted for $\epsilon$ ranging from $15^{\circ}$ to $55^{\circ}$ corresponding to the weak scattering regime at $327 \mathrm{MHz}$ which covers heliocentric distances between 0.26 and $0.82 \mathrm{AU}$.

For the present analysis and in order to obtain a uniform data set, it would be necessary to either choose sources of the same angular size or remove the effect of the finite source size by appropriately normalizing the data. The normalization was carried out using a least squares minimization to determine which of the Marians curves best fits the data for a given source. Since it is known that $1148-001$ is a good approximation to a point source, the observed values of $m$ of all other sources were multiplied by a factor equal to the difference between the best fit Marians curve for the given source and the best fit Marians curve for 1148-001, at the corresponding $\epsilon$. The best fit Marians curve for 1148-001 corresponds to that obtained for a source size of 10 mas.

The upper panel of Figure 3 shows, by filled blue dots, one example of the actual observations of $m$ as a function of heliocentric distance for the source 0003-003. The dashed red line is the Marians curve corresponding to a source size of 10 mas, while the dashed black line is the Marians curve which best fits the data for the source 0003-003. The middle panel of Figure 3 shows the same data after it has been normalized, as described above, to remove the effect of the finite source size. After normalizing all the observations in the above manner, we shortlist only those sources which had 


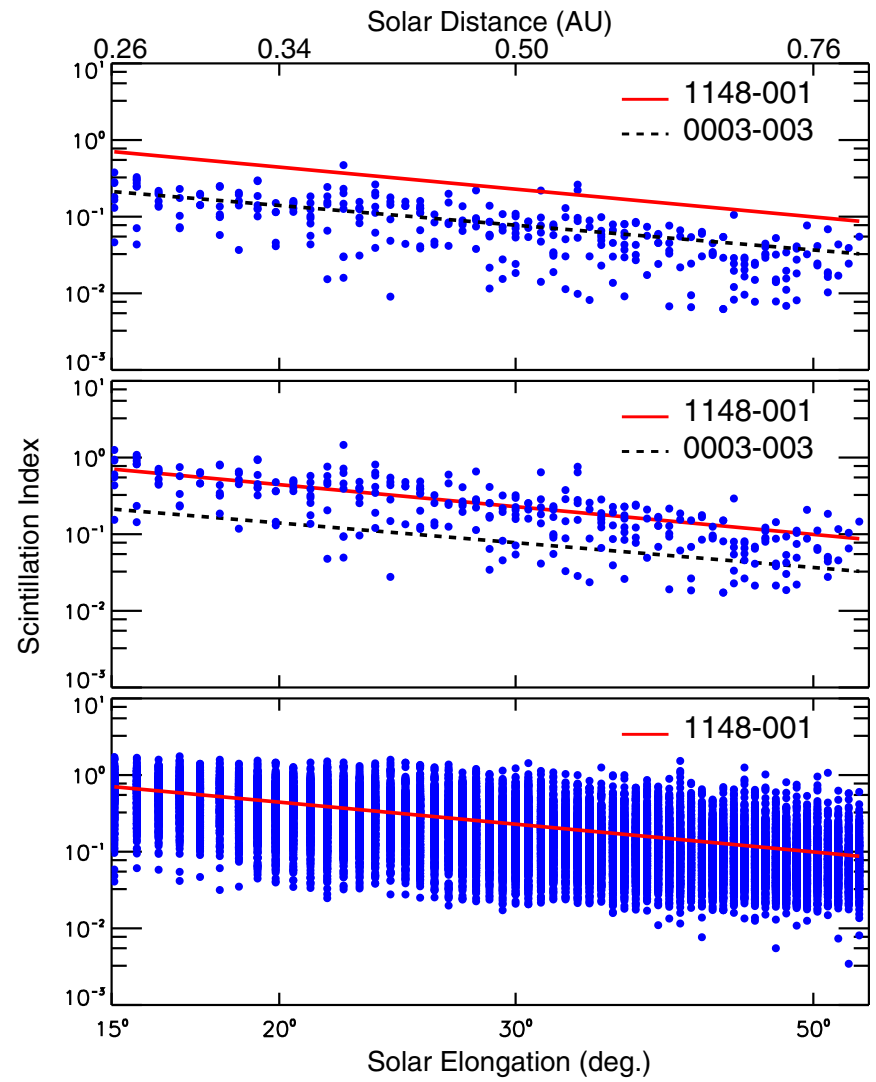

Figure 3. Upper panel shows, by filled blue dots, the actual measurements of normalized scintillation indices for the source 0003-003. The theoretically computed curve for $m$ using Marians' model (Marians 1975) for both 0003-003 (dotted black) and 1148-001 (red line) are overplotted. The middle panel shows the same two theoretical curves for sources 1148-001 and 0003-003 after the data of 0003-003 has been multiplied by a factor, determined from ratio of theoretical curves of 1148-001 and 0003-003 at each $\epsilon$, to remove the effects of source size. The lower panel shows the data for all 27 sources after being normalized to remove the source size effect. It can be seen that the data is well fitted to the theoretical curves of the source 1148-001.

(A color version of this figure is available in the online journal.)

at least 400 observations distributed uniformly over the entire range of heliocentric distances without any significant data gaps. Using this criteria, we finally shortlisted 27 sources for further analysis. The normalized points for all 27 sources are shown in the lowermost panel of Figure 3 and they fit the theoretical curve of the source 1148-001 very well. The right ascension and declination (J2000 epoch) of the 27 shortlisted radio sources are shown in Figure 4 by numbered open circles with the corresponding names of the sources (B1950 epoch) listed at the bottom of Figure 4 . The ecliptic radio sources in Figure 4 are those in the declination range $\pm 23^{\circ}$, while the non-ecliptic or high latitude sources lie above this range of declinations.

Using Equation (4), $\Delta N$ has been obtained at heliocentric distances in the range $0.26-0.82 \mathrm{AU}\left(55-175 R_{\odot}\right)$ from 1998 to 2008, using daily IPS measurements of $m$. In order to estimate the background solar wind density, we use values of the daily average solar wind density $(N)$ obtained from the Solar Wind Electron, Proton, and Alpha Monitor (SWEPAM) on board the $A C E$ spacecraft, covering the period from 1998 to 2008. However, $A C E$ density measurements are effectively at a distance of $1 \mathrm{AU}$. Thus, to estimate density at the locations, spread over distances of $0.26-0.82 \mathrm{AU}$, the measured $A C E$ densities at $1 \mathrm{AU}$ were extrapolated in the sunward direction using a background density model by Leblanc et al. (1998).

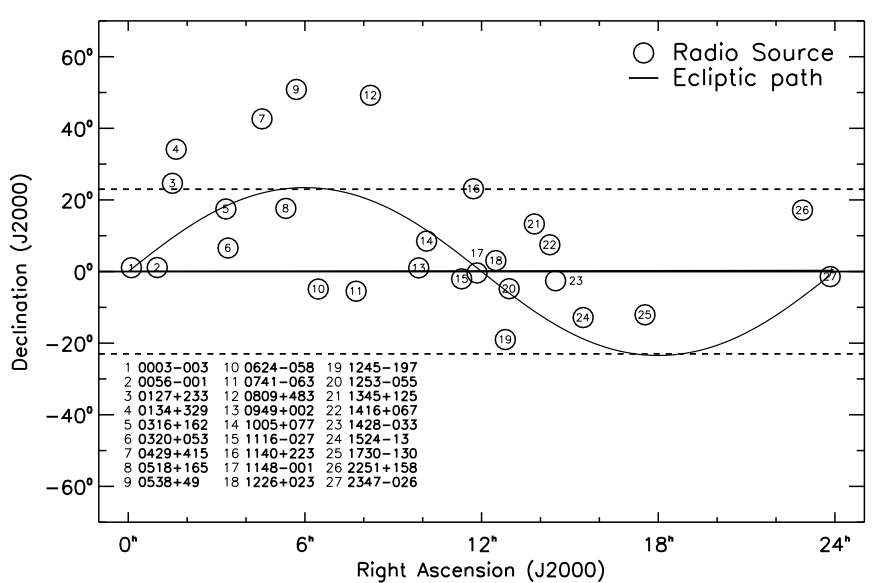

Figure 4. This figure shows the coordinates (R.A. and decl.) of the 27 selected radio sources by numbered open circles. The solid curve represents the path (R.A. and decl.) of the Sun. Each numbered source name is indicated at the bottom left of the figure.

According to this model, the background density, $N$ at $r$ (in units of $A U$ ) is given by

$$
N=7.2 r^{-2}+1.95 \times 10^{-3} r^{-4}+8.1 \times 10^{-7} r^{-6} \mathrm{~cm}^{-3} .
$$

This equation assumes a density of $7.2 \mathrm{~cm}^{-3}$ at $1 \mathrm{AU}$. In order to derive the background density at a given $r$, we use Equation (5) multiplied by $N(1 \mathrm{AU}) / 7.2$, where $N(1 \mathrm{AU})$ denotes the value of the density from the $A C E$ data. As discussed earlier, the $\triangle N$ is deduced from IPS measurements of $m$ using Equation (4). We compute $N$ by using near-Earth $A C E$ measurements that are contemporaneous with the measurement of $m$ and extrapolate it sunward to the heliocentric distance where $m$ is measured. For instance, let us consider the observation of the source 1148-001 in 1999 at an $\epsilon$ (heliocentric distance) of $15^{\circ}(0.26 \mathrm{AU})$. We use $A C E$ data at $1 \mathrm{AU}$ from 1999 and extrapolate it sunward to a heliocentric distance of $0.26 \mathrm{AU}$ to determine the appropriate $N$ to be used in Equation (1). The ratio of $\Delta N$ to $N$ gives $\epsilon_{N}$ (Equation (1)). As stated earlier, the $m$ of a given source is a function of both the distance of the LOS from the Sun and the source size, with ideal point-like radio sources giving an $m$ of $\approx 1$ at the start of the weak scattering regime which, at $327 \mathrm{MHz}$, is at a distance of approximately $0.2 \mathrm{AU}$. This is the reason that we can probe the solar wind at $327 \mathrm{MHz}$ over a distance range of $0.26-0.82 \mathrm{AU}\left(55-175 R_{\odot}\right)$.

\section{TEMPORAL AND SPATIAL BEHAVIOR OF $\epsilon_{N}$}

The upper panel of Figure 5 shows $\epsilon_{N}$ as a function of $r$ in the range 0.26 to $0.82 \mathrm{AU}$ and spanning the period 1998-2008. The solid blue and red dots represent the $\epsilon_{N}$ derived for ecliptic and non-ecliptic source observations, respectively, while their running averages at heliocentric distance intervals of $0.1 \mathrm{AU}$ are shown by large open circles with $1 \sigma$ error bars. The decline in $\epsilon_{N}$ is only $0.22 \%$. So it is quite apparent that $\epsilon_{N}$ is almost independent of heliocentric distance. The solid black line is a fit to the running averages of $\epsilon_{N}$, which emphasizes this trend. The Marians model, by assuming a spherically symmetric distribution of density fluctuations, ignores any latitudinal structure in the density fluctuations. IPS data of non-ecliptic sources are therefore likely to be affected by the latitudinal structure caused, for example, by polar coronal holes. So, the difference between ecliptic and non-ecliptic sources may be attributed to a bias caused by the effect of the solar wind latitudinal structure. 

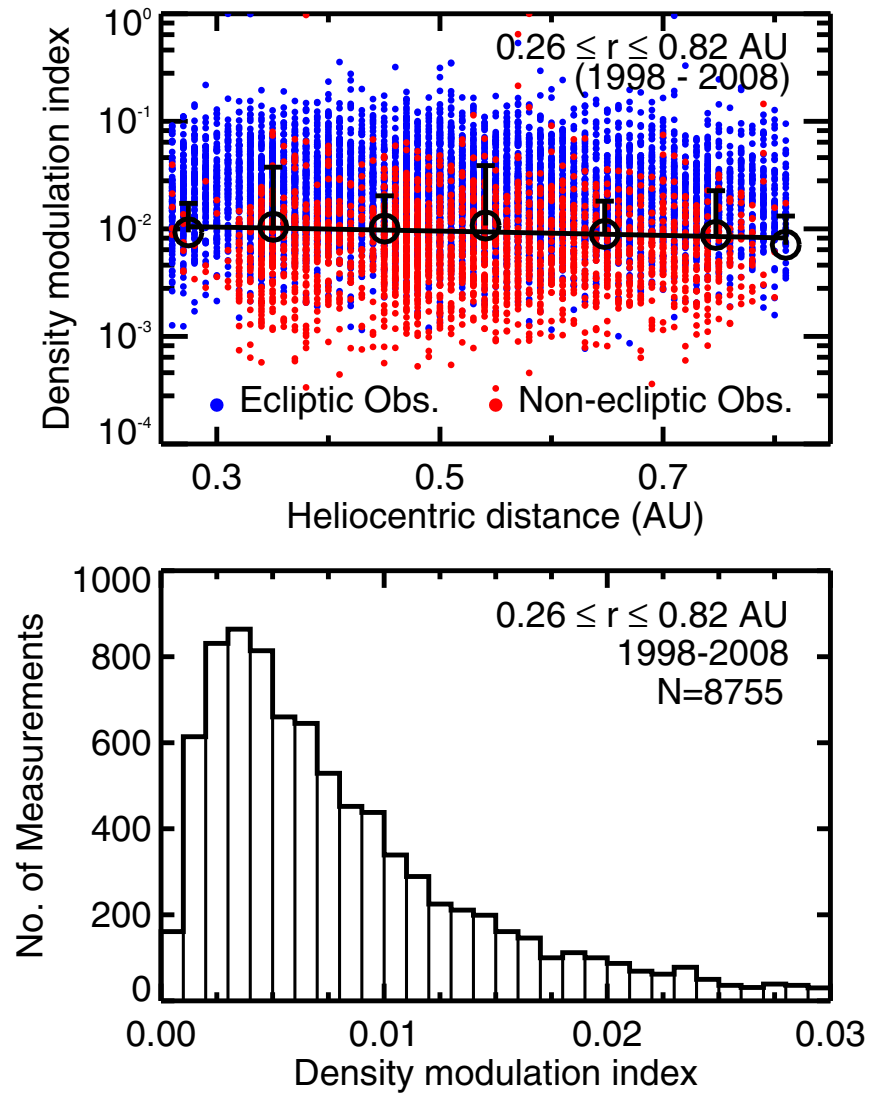

Figure 5. Upper panel: spatial variation of the density modulation index, $\epsilon_{N}$, of all 27 selected sources in the period from 1998 to 2008 . While the blue and red solid dots are the actual measurements of normalized modulation indices for ecliptic sources and non-ecliptic sources, respectively, the large open circles in black represent averages of all observation at intervals of $0.1 \mathrm{AU}$. The solid line is a fit to these average values. The lower panel shows a histogram of the $\epsilon_{N}$, with a median and mean of 0.006 and 0.01 , respectively.

(A color version of this figure is available in the online journal.)

Histograms of $\epsilon_{N}$ for the 27 selected sources used in the present analysis are shown in the lower panel of Figure 5. The total number of measurements are mentioned on the top right corner of Figure 5. An inspection of the histogram of $\epsilon_{N}$ shows that $0.001 \lesssim \epsilon_{N} \lesssim 0.02$ with a most probable value of 0.006 and a mean of 0.01 . These values are somewhat less than the values of $0.03 \lesssim \epsilon_{N} \lesssim 0.08$ reported using Wind spacecraft measurements of density fluctuations at $1 \mathrm{AU}$ (Spangler \& Spitler 2004). A modulation index $\epsilon_{N} \lesssim 0.1$ has been reported by Bavassano \& Bruno (1995) using measurements from the Helios 2 spacecraft between 0.03-1 AU. However, in both these papers, the data used only covered a limited time interval (albeit with a high sampling frequency of 45 minutes), whereas this study uses data for $11 \mathrm{yr}$, covering almost the entire solar cycle 23 (with a sampling frequency of one day).

Figure 6 shows the spatial variation of $\epsilon_{N}$ for IPS measurements of ecliptic (upper panel) and non-ecliptic sources (lower panel). The mean values of $\epsilon_{N}$ for ecliptic and non-ecliptic sources are $0.03 \pm 0.03$ and $0.01 \pm 0.02$, respectively, showing a slightly higher $\epsilon_{N}$ for the ecliptic sources. The decline in $\epsilon_{N}$ with heliocentric distance for the ecliptic and non-ecliptic sources are $0.7 \%$ and $0.25 \%$, respectively. So it is again clearly evident that $\epsilon_{N}$ is independent of heliocentric distance for both ecliptic and non-ecliptic sources.

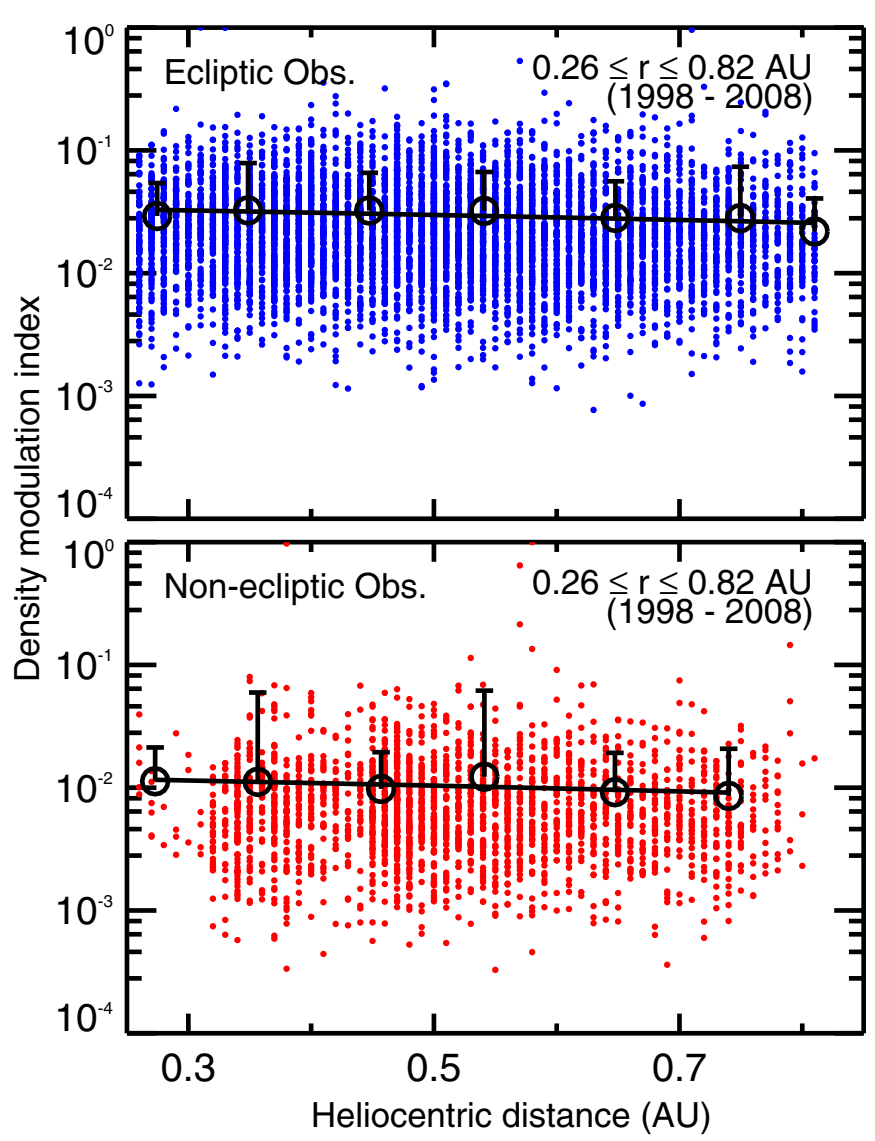

Figure 6. Upper panel: spatial variation of the $\epsilon_{N}$ for ecliptic sources in the period from 1998 to 2008 . The lower panel shows the spatial variation of the $\epsilon_{N}$ for non-ecliptic sources.

(A color version of this figure is available in the online journal.)

\subsection{Long-term Temporal Changes of $\epsilon_{N}$}

A study of the long-term changes in IPS measurements of $m$, a good proxy for solar wind microturbulence levels, has shown a systematic and steady decline in $m$ since $\approx 1995$ (Janardhan et al. 2011). One would therefore expect that electron density fluctuations, $\Delta N$, would also exhibit a similar decrease. In fact, a consistent decrease in electron density turbulence in regions of the inner heliosphere has been reported (Tokumaru et al. 2012) using IPS measurements from STEL. Using IPS measurements from the Ooty Radio Telescope, Manoharan (2012) also reported a declining trend of the density turbulence from 2004 to 2009 (see Figure 3 in Manoharan 2012). It is therefore of interest to see how $\epsilon_{N}$ varies in time during the period 1998-2008.

Figure 7 shows the temporal variation of $\epsilon_{N}$, covering the period 1998-2008, at heliocentric distances ranging from 0.26 to $0.82 \mathrm{AU}$. The blue solid dots are the derived density modulation indices while annual means of the modulation indices are shown by large red open circles with $1 \sigma$ error bars. The annual means of $\epsilon_{N}$ show a decline of $8 \%$ in $\epsilon_{N}$. This finding impacts our understanding of the steady temporal decline in solar wind dynamic pressure; we discuss this further in the next section.

\section{SUMMARY}

\subsection{Conclusions}

We have carried out an extensive survey of the density modulation index $\left(\epsilon_{N}\right)$ in the inner heliosphere using IPS observations at $327 \mathrm{MHz}$. We have used observations of 27 


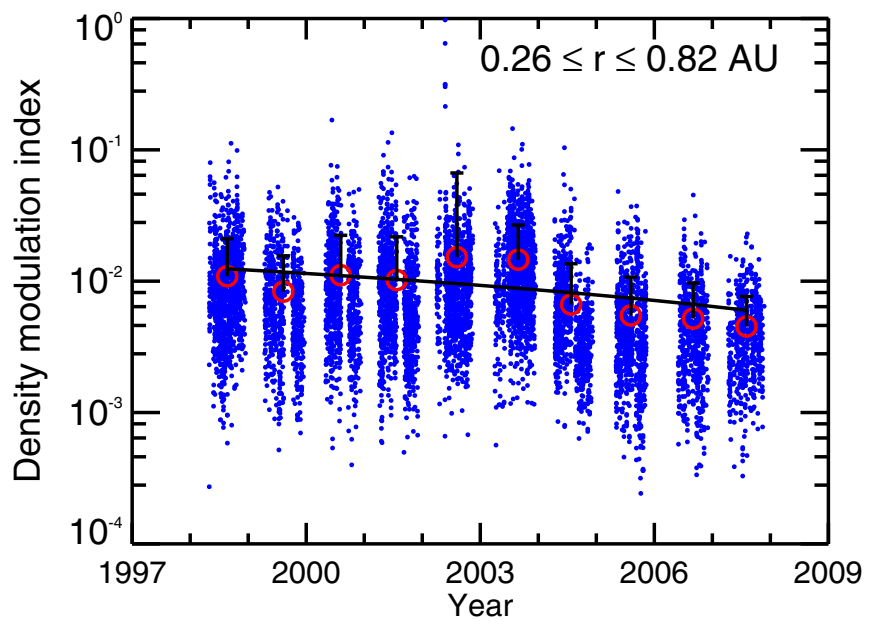

Figure 7. $\epsilon_{N}$ as function of time for the selected 27 sources at heliocentric distances of $0.26-0.82 \mathrm{AU}$. While the blue solid dots are the actual measurements of $\epsilon_{N}$, the large open circles in red represent annual means. The solid curve is a linear fit to annual means of $\epsilon_{N}$.

(A color version of this figure is available in the online journal.)

sources spanning the heliocentric distance range 0.26-0.82 AU for the period 1998-2008. One of the broad conclusions of our study is that $\epsilon_{N} \approx 0.01$ and is roughly constant with heliocentric distance. Our result shows the typical amplitudes of density modulation index are low, of the order of $0.1 \%-2 \%$, and these values are somewhat lower than the values of $3 \%-8 \%$ reported by Spangler \& Spitler (2004). It may be noted, however, that Spangler \& Spitler (2004) have used only nearEarth observations, whereas our observations span a heliocentric distance range of 0.26-0.82 AU. Earlier measurements (Tu \& Marsch 1994 and Bavassano \& Bruno 1995) of $\epsilon_{N}$ from Helios data at heliocentric distances between 0.3 and 0.5 AU have found $5 \% \lesssim \epsilon_{N} \lesssim 20 \%$.

Our result of $\epsilon_{N}$ being independent of heliocentric distance agrees with those proposed by Woo et al. (1995) for the slow solar wind. Using Ulysses time delay measurements, Woo et al. (1995) have shown that the relative density fluctuations obtained over a period of $5 \mathrm{hr}$ for the slow solar wind $\left(\leqslant 250 \mathrm{~km} \mathrm{~s}^{-1}\right)$ in the distance range from 0.03 to $1 \mathrm{AU}$ is independent of heliocentric distance.

The long-term temporal variation of the relative density fluctuations over heliocentric distances of $0.26-0.82$ AU have shown a decline of 8\% during the period 1998-2008.

\subsection{Discussion}

We now comment on the implications of our results on some of the problems we have outlined in the Introduction.

1. The scintillation levels in the inner heliosphere (which are $\propto \Delta \mathrm{N})$ have been shown to be declining monotonically since $\approx 1995$ (Janardhan et al. 2011; Tokumaru et al. 2012). Assuming that $\Delta N \propto$ the background density $N$, this has prompted speculations about a steady temporal decline in the pressure exerted by the bulk solar wind on Earth's magnetosphere. McComas et al. (2013) have calculated the canonical standoff distance of bow shock nose of Earth's magnetosphere, which is about 11 Earth radii $\left(R_{E}\right)$ for the period 2009-2013 compared to about $10 R_{E}$ for the period 1974-1994. According to these authors, this change is in view of the observed decline in solar wind dynamic pressure from $\sim 2.4 \mathrm{nPa}(1974-1994)$ to $\sim 1.4 \mathrm{nPa}$ (2009-2013).
However, these need to be revisited in light of our findings of a small, but discernible, steady decrease in $\epsilon_{N} \equiv \Delta N / N$ with time.

Furthermore, if there is a linear relationship between the relative density fluctuations and the magnetic field fluctuations (Spangler \& Spitler 2004), it would imply that the magnetic field fluctuations also decline steadily over period 1998-2008. So it appears reasonable to conclude that the decrease in density fluctuations is connected to the unusual solar magnetic activity during the long deep solar minimum at the end of the solar cycle 23 . It has been shown that both solar polar fields and the level of turbulent density fluctuations $(\Delta N)$ have decreased monotonically since around 1995 (Janardhan et al. 2010, 2011; Bisoi et al. 2014).

2. We note that the IPS technique used in this work to infer density fluctuations is sensitive to spatial scales of 50-1000 km (Pramesh Rao et al. 1974; Coles \& Filice 1985; Fallows et al. 2008). It is worth examining how these scales relate to the dissipation scale of the turbulent cascade (often referred to as the inner scale). If the length scales probed by the IPS technique are in the inertial range, it is reasonable to presume that the magnetic field is frozen in, and the density fluctuations can then be taken as a proxy for magnetic field fluctuations (e.g., Spangler 2002). We note, however, that the flux-freezing concept might not hold for turbulent fluids (e.g., Lazarian \& Vishniac 1999). In order to investigate this issue, we consider three popular inner scale prescriptions. One prescription for the inner scale assumes that the turbulent wave spectrum is dissipated due to ion cyclotron resonance, and the inner scale is the ion inertial scale (Coles \& Harmon 1989). In this case, the inner scale $\left(l_{i}\right)$ is given as a function of heliocentric distance $r$ by

$$
l_{i}=684 n_{e}(r)^{-1 / 2} \mathrm{~km},
$$

where $n_{e}$ is the number density in $\mathrm{cm}^{-3}$. A second prescription identifies the inner scale with the proton gyroradius (Bale et al. 2005; Alexandrova et al. 2012). In this case the inner scale is given by

$$
l_{i}(r)=1.02 \times 10^{2} \mu^{1 / 2} T_{i}^{1 / 2} B(r)^{-1} \mathrm{~cm},
$$

where $\mu\left(\equiv m_{p} / m_{e}\right)$ is the proton to electron mass ratio, $T_{i}$ is the proton temperature in $\mathrm{eV}$, and $B$ is the Parker spiral magnetic field in the ecliptic plane (Williams 1995). However, recent work seems to suggest that the dissipation could occur at scales as small as the electron gyroradius (Alexandrova et al. 2012; Sahraoui et al. 2013). The third prescription we therefore consider is one where the inner scale is taken to be equal to the electron gyroradius and is given by

$$
l_{i}(r)=2.38 \times T_{e}^{1 / 2} B(r)^{-1} \mathrm{~cm},
$$

where $T_{e}$ is the electron temperature in $\mathrm{eV}$. The inner scales using these three prescriptions (Equations (6)-(8)) are shown in Figure 8 as a function of heliocentric distance. The gray band denotes the range of length scales $(\approx 50-1000 \mathrm{~km})$ to which the IPS technique is sensitive. As explained in the caption of Figure 8, we use electron and proton temperatures of $10^{5} \mathrm{~K}$ in order to compute the proton and electron gyro radii respectively. The magnetic field is 


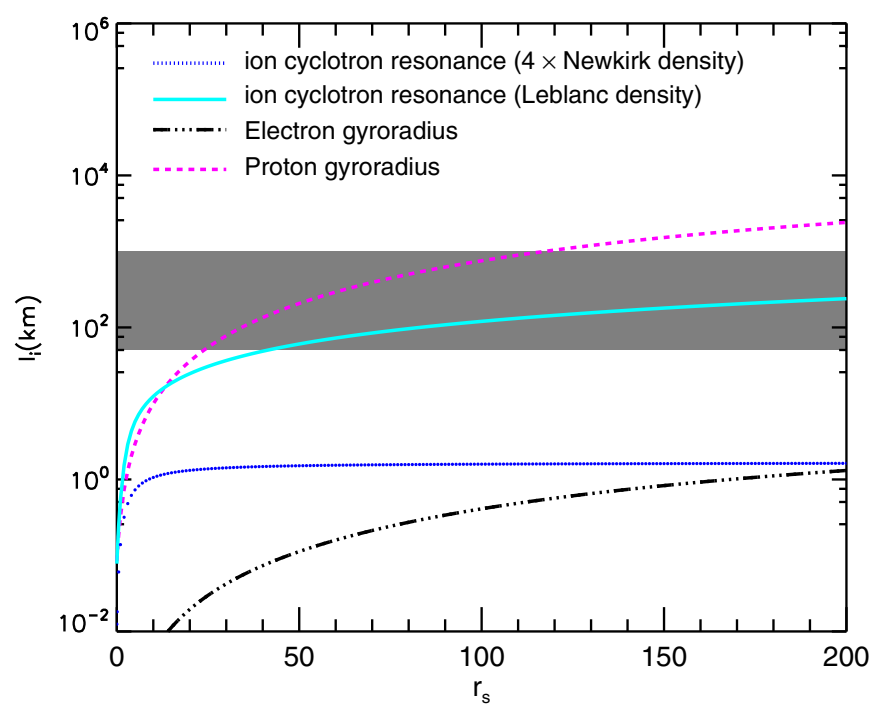

Figure 8. Inner scale $l_{i}$ in kilometers as a function of heliocentric distance in units of solar radii $\left(r_{s}\right)$. The dashed line shows the proton gyroradius using a proton temperature of $10^{5} \mathrm{~K}$. The solid and dotted lines shows the inner scale governed by ion cyclotron resonance using the Leblanc et al. density model and the fourfold Newkirk density model, respectively. The dot-dashed line shows the electron gyroradius using an electron temperature of $10^{5} \mathrm{~K}$. The light gray region denotes the range of spatial scales to which IPS observations are sensitive. (A color version of this figure is available in the online journal.)

taken to be a standard Parker spiral (Williams 1995). In order to compute the inner scale using Equation (6), we need a density model. We have used two representative density models - the Leblanc density model (Leblanc et al. 1998) and the fourfold Newkirk density model (Newkirk 1961). If the length scales probed by the IPS technique (denoted by the gray band in Figure 8) are larger than the inner scale, we can conclude that the density fluctuations discussed in this paper lie in the inertial range of the turbulent spectrum. From Figure 8, it is evident that this is the case all the way from the Sun to the Earth only if the inner scale is the electron gyroradius, or if it is due to proton cyclotron resonance, and the density is given by the fourfold Newkirk model. On the other hand, if the inner scale is given by the proton gyroradius, or if the inner scale is due to proton cyclotron resonance and the density model is given by the Leblanc et al. (1998) prescription, the density fluctuations probed by the IPS technique are probably smaller than the dissipation scale for heliocentric distances beyond $30-40 R_{\odot}$.

3. In order to account for the magnitude of cosmic ray Forbush decreases observed at the Earth, Subramanian et al. (2009) and Arunbabu et al. (2013) deduce that the level of magnetic field turbulence in the sheath region ahead of Earth-directed CMEs ranges from a few to a few tens of percent. The magnetic field turbulence level is often taken to be a proxy for $\epsilon_{N}$ (Spangler 2002). Generally, the turbulence level in the sheath region would be expected to be somewhat higher than (but not very different from) its value in the quiescent solar wind. The results of this paper regarding the magnitude of $\epsilon_{N}$ in the quiescent solar wind are thus broadly consistent with the deductions of Subramanian et al. (2009) and Arunbabu et al. (2013) regarding the magnetic field turbulence level.

4. Reid \& Kontar (2010) have argued that the modulation index $\epsilon_{N}$ needs to be around 10\% near the Earth and be proportional to $R^{0.25}$ (where $R$ is the heliocentric distance) in order to account for the Earthward transport of electron beams produced in solar flares. However, we find that the modulation index shows no change with increasing heliocentric distance, and that its value near the Earth is considerably smaller than $10 \%$.

IPS observations were carried out under the solar wind program of STEL, Japan. We thank the ACE SWEPAM instrument team and the $A C E$ Science Center for providing the $A C E$ data available in the public domain via World Wide Web. We thank the reviewer for constructive comments which have improved the paper significantly.

\section{REFERENCES}

Alexandrova, O., Lacombe, C., Mangeney, A., Grappin, R., \& Maksimovic, M. 2012, ApJ, 760, 121

Ananthakrishnan, S., Coles, W. A., \& Kaufman, J. J. 1980, JGR, 85, 6025

Ananthakrishnan, S., Rao, A. P., \& Bhandari, S. M. 1975, Ap\&SS, 37, 275

Arunbabu, K. P., Antia, H. M., Dugad, S. R., et al. 2013, A\&A, 555, A139

Asai, K., Kojima, M., Tokumaru, M., et al. 1998, JGR, 103, 1991

Bale, S. D., Kellogg, P. J., Mozer, F. S., Horbury, T. S., \& Reme, H. 2005, PhRvL, 94, 215002

Bastian, T. S. 1994, ApJ, 426, 774

Bavassano, B., \& Bruno, R. 1995, JGR, 100, 9475

Bird, M. K., Janardhan, P., Efimov, A. I., et al. 2003, in AIP Conf. Proc. Vol. 679, Solar Wind Ten, ed. M. Velli, R. Bruno, F. Malara, \& B. Bucci (Mel;ville, NY: AIP), 465

Bisoi, S. K., Janardhan, P., Chakrabarty, D., Ananthakrishnan, S., \& Divekar, A. 2014, SoPh, 289, 41

Bourgois, G. 1969, A\&A, 2, 209

Bourgois, G., \& Creynet, C. 1972, A\&A, 21, 25

Bruno, R., \& Carbone, V. 2005, LRSP, 2, 4

Carbone, V., Marino, R., Sorriso-Valvo, L., Noullez, A., \& Bruno, R. 2009, PhRvL, 103, 061102

Clark, B. G., Kellermann, K. I., Bare, C. C., Cohen, M. H., \& Jauncey, D. L. 1968, ApJ, 153, 705

Clarke, R. W., Broten, N. W., Legg, T. H., Locke, J. L., \& Yen, J. L. 1969, MNRAS, 146, 381

Coles, W. A. 1978, SSRv, 21, 411

Coles, W. A., \& Filice, J. P. 1985, JGR, 90, 5082

Coles, W. A., \& Harmon, J. K. 1989, ApJ, 337, 1023

Efimov, A. I., Rudash, V. K., Bird, M. K., et al. 2000, AdSpR, 26, 785

Fallows, R. A., Breen, A. R., \& Dorrian, G. D. 2008, AnGeo, 26, 2229

Goldstein, M. L., Roberts, D. A., \& Matthaeus, W. H. 1995, ARA\&A, 33, 283

Hewish, A., Tappin, S. J., \& Gapper, G. R. 1985, Natur, 314, 137

Hnat, B., Chapman, S. C., \& Rowlands, G. 2005, PhRvL, 94, 204502

Huddleston, D. E., Woo, R., \& Neugebauer, M. 1995, JGR, 100, 19951

Janardhan, P., \& Alurkar, S. K. 1993, A\&A, 269, 119

Janardhan, P., Alurkar, S. K., Bobra, A. D., \& Slee, O. B. 1991, AuJPh, 44, 565

Janardhan, P., Alurkar, S. K., Bobra, A. D., Slee, O. B., \& Waldron, D. 1992, AuJPh, 45, 115

Janardhan, P., Balasubramanian, V., Ananthakrishnan, S., et al. 1996, SoPh, 166,379

Janardhan, P., Bisoi, S. K., Ananthakrishnan, S., Tokumaru, M., \& Fujiki, K. 2011, GeoRL, 38, L20108

Janardhan, P., Bisoi, S. K., \& Gosain, S. 2010, SoPh, 267, 267

Jian, L. K., Russell, C. T., \& Luhmann, J. G. 2011, SoPh, 274, 321

Kojima, M., \& Kakinuma, T. 1990, SSRv, 53, 173

Lazarian, A., \& Vishniac, E. T. 1999, ApJ, 517, 700

Leblanc, Y., Dulk, G. A., \& Bougeret, J.-L. 1998, SoPh, 183, 165

Manoharan, P. K. 2012, ApJ, 751, 128

Marians, M. 1975, RaSc, 10, 115

Marsch, E. 2006, LRSP, 3, 1

Marsch, E., \& Tu, C.-Y. 1990, JGR, 95, 11945

McComas, D. J., Angold, N., Elliott, H. A., et al. 2013, ApJ, 779, 2

McComas, D. J., Ebert, R. W., Elliott, H. A., et al. 2008, GeoRL, 35, L18103

Milne, R. G. 1976, AuJPh, 29, 201

Newkirk, G., Jr. 1961, ApJ, 133, 983

Pramesh Rao, A., Bhandari, S. M., \& Ananthakrishnan, S. 1974, AuJPh, 27, 105

Reid, H. A. S., \& Kontar, E. P. 2010, ApJ, 721, 864

Sahraoui, F., Huang, S. Y., Belmont, G., et al. 2013, ApJ, 777, 15

Shaikh, D., \& Zank, G. P. 2010, MNRAS, 402, 362

Spangler, S. R. 2002, ApJ, 576, 997 
Spangler, S. R. 2009, SSRv, 143, 277

Spangler, S. R., \& Spitler, L. G. 2004, PhP1, 11, 1969

Stone, E. C., Frandsen, A. M., Mewaldt, R. A., et al. 1998, SSRv, 86, 1

Subramanian, P., Antia, H. M., Dugad, S. R., et al. 2009, A\&A, 494, 1107

Subramanian, P., \& Cairns, I. 2011, JGRA, 116, 3104

Swarup, G. 1977, J. Sci. Ind. Res., 36, 569

Thejappa, G., \& MacDowall, R. J. 2008, ApJ, 676, 1338

Tokumaru, M., Kojima, M., \& Fujiki, K. 2012, JGRA, 117, 6108
Tu, C.-Y., \& Marsch, E. 1994, JGR, 99, 21481

Tu, C.-Y., \& Marsch, E. 1995, SSRv, 73, 1

Venugopal, V. R., Ananthakrishnan, S., Swarup, G., Pynzar, A. V., \& Udaltsov, V. A. 1985, MNRAS, 215, 685

Williams, L. L. 1995, ApJ, 453, 953

Woo, R., Armstrong, J. W., Bird, M. K., \& Patzold, M. 1995, GeoRL, 22, 329

Yamauchi, Y., Tokumaru, M., Kojima, M., Manoharan, P. K., \& Esser, R. 1998, JGR, 103, 6571 